\title{
Corrosion protection of steel by electropolymerization of $o$-chloroaniline and $o$-aminobenzoic acid in the presence of Tween 80 surfactant
}

\author{
B.A. Abd-El-Nabey, ${ }^{1} *$ O.A. Abdullatef ${ }^{2}$ and R.M. Salman ${ }^{1}$ \\ ${ }^{1}$ Faculty of Science, Chemistry Department, Alexandria University, P.O.Box 426, \\ Alexandria 21321, Egypt \\ ${ }^{2}$ Pharos University, Faculty of Pharmacy, P.O.Box 3231, Alexandria, Egypt \\ *E-mail: beshirabdelnaby2015@gmail.com
}

\begin{abstract}
The conducting polymers, poly-o-chloroaniline and poly-o-aminobenzoic acid have been synthesized from their monomers on steel surface by cyclic voltammetry method in aqueous oxalic acid solution containing different concentrations of Tween 80 surfactant. The polymers were characterized by scanning electron microscope SEM. Potentiodynamic polarization and electrochemical impedance spectroscopy EIS techniques have been used to investigate the corrosion behavior of the polymer coated steel electrodes in $0.5 \mathrm{M} \mathrm{H}_{2} \mathrm{SO}_{4}$ solution. Effect of the surfactant on the electropolymerization process and the protection efficiency of the polymerized coat have been studied. Effect of substituent in the molecule of the monomer on the protection efficiency of the polymerized coat has been also studied.
\end{abstract}

Keywords: conducting polymers; cyclic voltammetry; corrosion; surfactant.

Received: October 28, 2017. Revised: December 28, 2017. Published: January 22, 2018

doi: $10.17675 / 2305-6894-2018-7-1-6$

\section{Introduction}

Carbon steel is the most important metal used in industry due to its excellent strong strength, low cost, ability to be recycled and good mechanical workability. However, steels have a major disadvantage in that they have low corrosion resistance. Our group of research is interesting in corrosion control of steel using inhibitors and coatings. Five categories of inhibitors were investigated in our laboratory using chemical and electrochemical techniques to clarify and discuss their inhibition characteristics and adsorption mechanisms on the steel surface in acid solutions. These five categories are: 1) simple and synthetic organic compounds [1,2]; 2) chelating agents [2];3) plant extracts [3]; 4) surfactants [4] and 5) silver nanoparticles [5].

Surfactants have a great influence on the growth of crystals and have the ability to alter crystallization kinetics [6]. This property can be efficiently used to produce coatings with desirable properties. A conversion coating is the most effective method to prevent corrosion of steel in a wide range of applications [7-9]. Recently, we investigated the 
effect of Tween 80 surfactant on the corrosion resistance of Zn-phosphated steel using potentiodynamic polarization, electrochemical impedance spectroscopy, SEM and EDX analysis techniques [10]. Electrochemical results indicated that the presence of $0.01 \mathrm{M}$ Tween 80 in the coating solution caused a decrease in the porosity and an increase of the protection efficiency of the coat up to $90 \%$. The results proved that the coating process was controlled by adsorption of the surfactant molecules on the steel surface. SEM studies and EDX analysis gave very good support to the electrochemical data.

Several research groups studied the electrochemical synthesis of conducting polymer coatings on oxidizable metals for corrosion protection purposes [11-17]. We studied the effect of Tween 80 surfactant on the electropolymerization and corrosion performance of polyaniline on steel [18]. The cyclic voltametric results indicated that the presence of Tween 80 surfactant in the polymerization medium of polyaniline leads to the inhibition of the oxidation of each of steel and aniline. The potentiodynamic polarization and electrochemical impedance results of polyaniline coated steel in $0.5 \mathrm{M} \mathrm{H}_{2} \mathrm{SO}_{4}$ solution indicated that the protection efficiency of the polymer coat to corrosion increase to about $80 \%$ at $0.05 \mathrm{M}$ Tween 80 .

The electrochemical polymerization of aniline and metanilic acid in aqueous perchloric acid leads to the formation of a copolymer similar to that obtained by direct sulfonation of polyaniline. Characterization by electrochemical methods indicated the presence of polyaniline linear chains in which metanilic acid units were inserted as spacers [19].

In this work, two polyaniline derivatives conducting polymers have been synthesized from $o$-chloroaniline and $o$-aminobenzoic acid monomers on carbon steel surface by cyclic voltammetry method in aqueous oxalic acid solution containing different concentrations of Tween 80 surfactant. Characterization of coatings was carried out by scanning electron microscope SEM. Corrosion behavior of the polymer coated steel electrodes was investigated in $0.5 \mathrm{M} \mathrm{H}_{2} \mathrm{SO}_{4}$ solution using potentiodynamic polarization and electrochemical impedance spectroscopy techniques. The aim of the work is to: 1) throw more light on the role of the surfactant in the electropolymerization process and 2) investigate the effect of substituent in the monomer molecule on the formation of the polymerized coat.

\section{Experimental}

\subsection{Chemicals and Apparatus}

$o$-Chloroaniline, $o$-aminobenzoic acid, oxalic acid and sulfuric acid used in this study were all purchased from Sigma Chemical company and used without any further purifications. All electrochemical polymerization experiments were performed in aqueous solution of aniline derivatives. Electrosynthesis and electrochemical studies were carried out in a three electrode system with steel as working electrode, platinum sheet was used as counter electrode and saturated calomel electrode (SCE) as reference electrode. The working 
electrode was constructed from a steel rod of $0.6 \mathrm{~cm}$ diameter with chemical composition (wt\%): C 0.21; S 0.04; Mn 2.5; P 0.04; Si 0.35; balance Fe. Prior to each experiment the working electrode was polished using a series of emery papers (300, 800 and 1000 grades) and cleaned with distilled water and ethanol and then dried in air. ACM 604 Potentiostat instrument were used for electrochemical synthesis and corrosion performance studies. The analysis of impedance spectra were performed by ZSimpWin program by fitting the experimental data to the appropriate equivalent circuit.

\subsection{Synthesis of aniline derivatives polymers}

The poly( $o$-chloroaniline) and poly( $o$-aminobenzoic acid) coatings were synthesized by electropolymerization of $o$-chloroaniline and $o$-aminobenzoic acid on steel substrate from aqueous solutions containing $0.3 \mathrm{M}$ oxalic acid in the absence and presence of different concentrations of Tween 80 surfactant using cyclic voltammetry technique in the potential range 300 to $1200 \mathrm{mV} v \mathrm{SCE}$ at scan rate $10 \mathrm{mV} \mathrm{s}^{-1}$ over 10 cycles. Prior to the cyclic voltammetry scan, linear sweep voltametry from -500 to $+300 \mathrm{mV} v s \mathrm{SCE}$ at $4 \mathrm{mV} / \mathrm{s}$ was carried out in order to passivate the steel substrate. After electrodeposition of polyaniline derivatives on steel substrate, the polymer coated substrates were removed from the electropolymerization medium and rinsed with double distilled water to remove monomer molecules and dried in air.

\subsection{Characterization of polyaniline derivatives coats}

The morphologies of the coat polymers were investigated by Scanning Electron Microscope SEM using a JOEL instrument.

\subsection{Corrosion performance measurements}

Corrosion performance of coated and uncoated steel electrodes were carried out in aqueous solutions of $0.5 \mathrm{M} \mathrm{H}_{2} \mathrm{SO}_{4}$ by potentiodynamic polarization and electrochemical impedance spectroscopy (EIS) measurements. The polarization curves were recorded by changing the potential between -300 and $+300 \mathrm{mV}$ around the rest potential with scan rate $30 \mathrm{mV} \mathrm{s}^{-1}$. The frequency range of impedance technique is 30000 to $0.1 \mathrm{~Hz}$ using $\mathrm{AC}$ with amplitude of $5 \mathrm{mV}$.

\subsection{Preparation of Solutions}

The acids used in this study are of analytical grade (Aldrich Chemicals) $98 \% \mathrm{H}_{2} \mathrm{SO}_{4}$ and 99\% oxalic acid. The $o$-chloroaniline $(99 \%)$ and $o$-aminobenzoic acid $(99 \%)$ were used without purification. TW 80 surfactant was obtained from Alpha Chemika with molecular weight $1310 \mathrm{~g} / \mathrm{mol}$ and its molecular structure is given in Figure 1. 


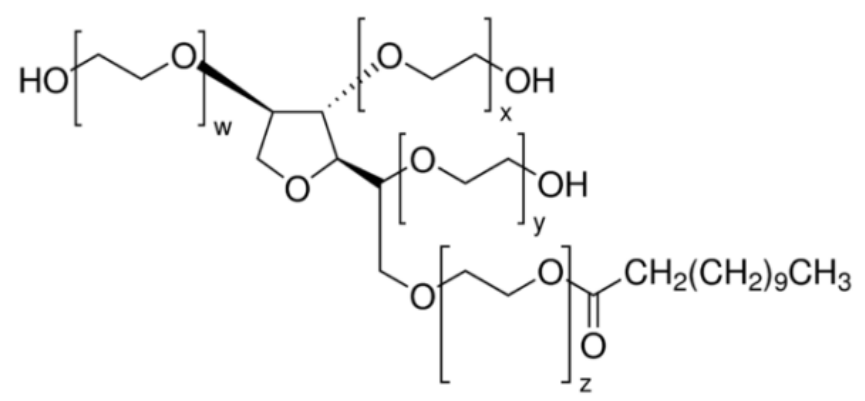

Figure 1. The molecular structure of Tween 80 .

Stock solutions $5 \mathrm{M} \mathrm{H}_{2} \mathrm{SO}_{4}, 1 \mathrm{M}(\mathrm{COOH})_{2}$ and $0.1 \mathrm{M}$ TW 80 surfactant were used to prepare test solutions. A stock solution of $o$-aminobenzoic acid monomer of $0.075 \mathrm{M}$ was prepared in dimethyl formamide (DMF). The test solution used for electropolymerization process of $o$-chloroaniline is prepared by adding $1.05 \mathrm{ml} o$-chloroaniline $(99 \%)$ to $30 \mathrm{ml}$ oxalic acid $(1 \mathrm{M})$ and a certain volume of TW $80(0.1 \mathrm{M})$ and diluted by doubly distilled water to $100 \mathrm{ml}$. The test solution used for electropolymerization process of $o$-aminobenzoic acid is prepared by adding $6.7 \mathrm{ml} o$-aminobenzoic acid $(0.075 \mathrm{M})$ to $3.3 \mathrm{ml} \mathrm{DMF}$ (i.e. $10 \% \mathrm{v} / \mathrm{v} \mathrm{DMF})$ to $30 \mathrm{ml}$ oxalic acid $(1 \mathrm{M})$ and certain volume of TW $80(0.1 \mathrm{M})$ and diluted by doubly distilled water to $100 \mathrm{ml}$.

The anticorrosion properties of the formed coats were evaluated in $0.5 \mathrm{M} \mathrm{H}_{2} \mathrm{SO}_{4}$ as a corrosive medium, by adding $10 \mathrm{ml}$ of $5 \mathrm{M} \mathrm{H}_{2} \mathrm{SO}_{4}$ and complete the volume to $100 \mathrm{ml}$ with double distilled water.

\section{Results and Discussion}

\section{A. Effect of Tween 80 surfactant on the Oxidation of Steel and the Monomers}

a. Electropolymerization of the monomers in presence of Tween 80

Cyclic voltammograms were recorded for both $0.1 \mathrm{M} o$-chloroaniline and $0.005 \mathrm{M} o$ aminobenzoic acid in $0.3 \mathrm{M}$ oxalic acid. The cyclic voltammetry were carried out in the presence of TW 80 as surfactant for 10 cycles at scan rate $10 \mathrm{mV} / \mathrm{sec}$. The first cycle voltammograms of steel during electropolymerization of each of $o$-chloroaniline (Figure $2 \mathrm{a}$ ) and $o$-aminobenzoic acid (Figure $2 \mathrm{~b}$ ) in $0.3 \mathrm{M}$ oxalic acid, in absence of the surfactant, are characterized by a large peak at $125 \mathrm{mV}$ and $150 \mathrm{mV}$ respectively. This peak mainly corresponds to the oxidation of steel. The dependence of potential of this peak on the molecular structure of the monomer molecule can be discussed on the basis that the monomer acts as inhibitor for dissolution of steel, and the kind of the substituted group in the monomer molecule affects its adsorbability at the steel/solution interface. However, in presence of TW 80 surfactant, the potential of this peak shifts to more anodic values and its height decreases with increasing concentration of the surfactant. This behavior can be attributed to the adsorption of TW 80 molecules at the steel/solution interface which inhibit the oxidation of steel. 


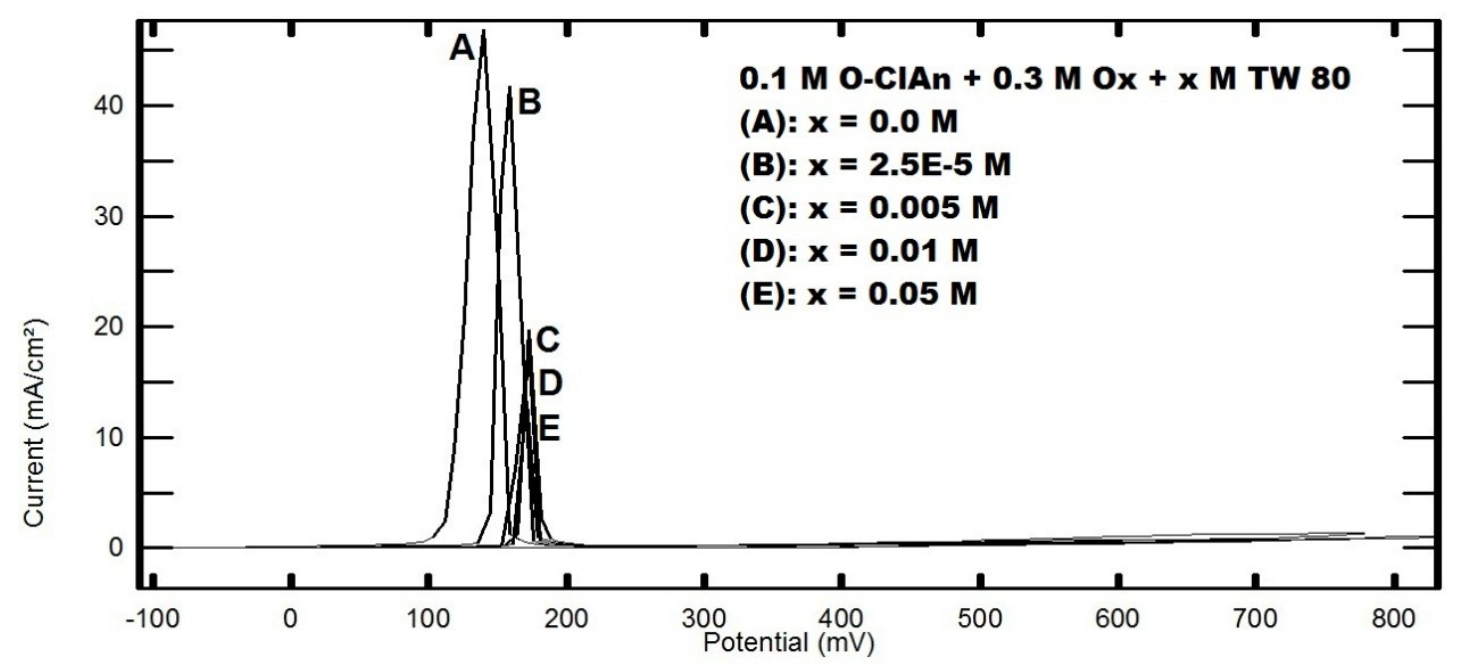

Figure 2a. The first cyclic voltammogram of steel during electropolymerization of $0.1 \mathrm{M}$ $o$-chloroaniline in $0.3 \mathrm{M}$ oxalic acid in absence and presence of different concentrations of TW 80 .

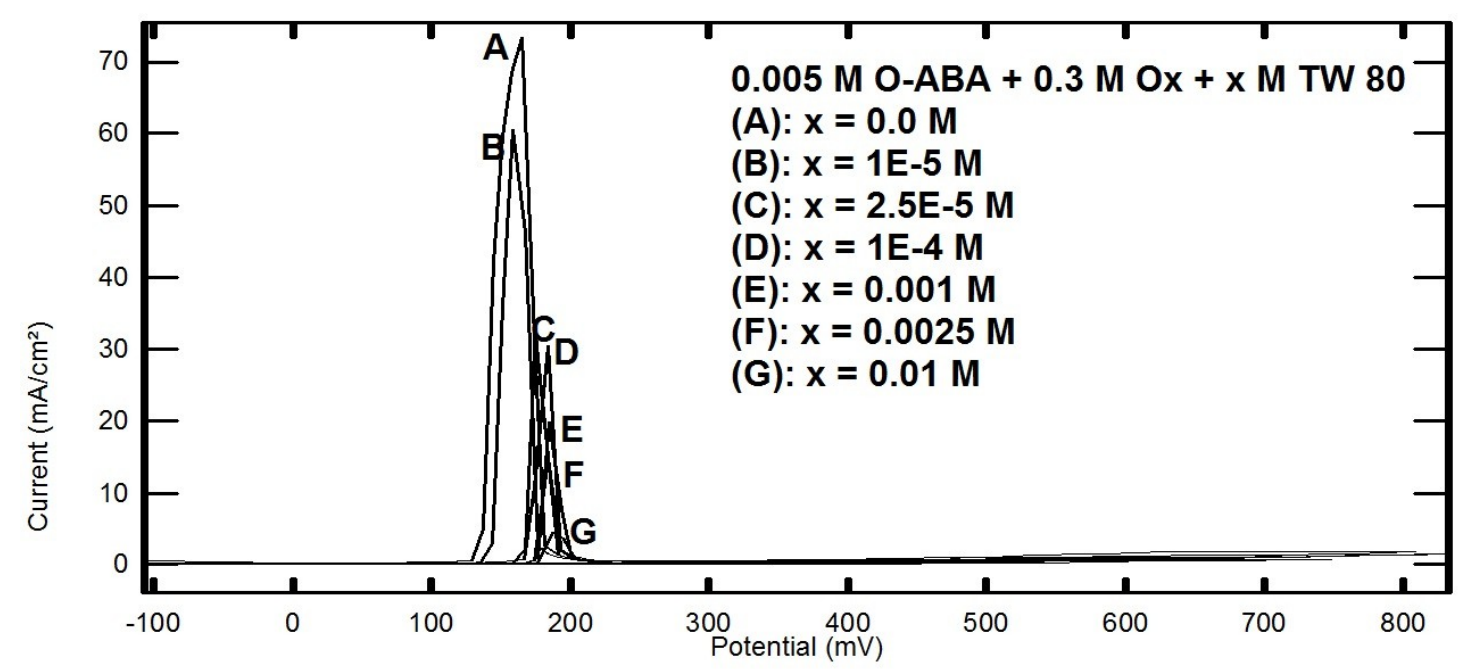

Figure 2b. The first cyclic voltammogram of steel during electroploymerization of $0.005 \mathrm{M}$ $o$-aminobenzoic acid in $0.3 \mathrm{M}$ oxalic acid in absence and presence of different concentrations of TW 80.

Figure 3a,b represents the third cyclic voltammogram of steel during the electropolymerization of each of $o$-chloroaniline and $o$-aminobenzoic acid in $0.3 \mathrm{M}$ oxalic acid in absence and presence of TW 80. 


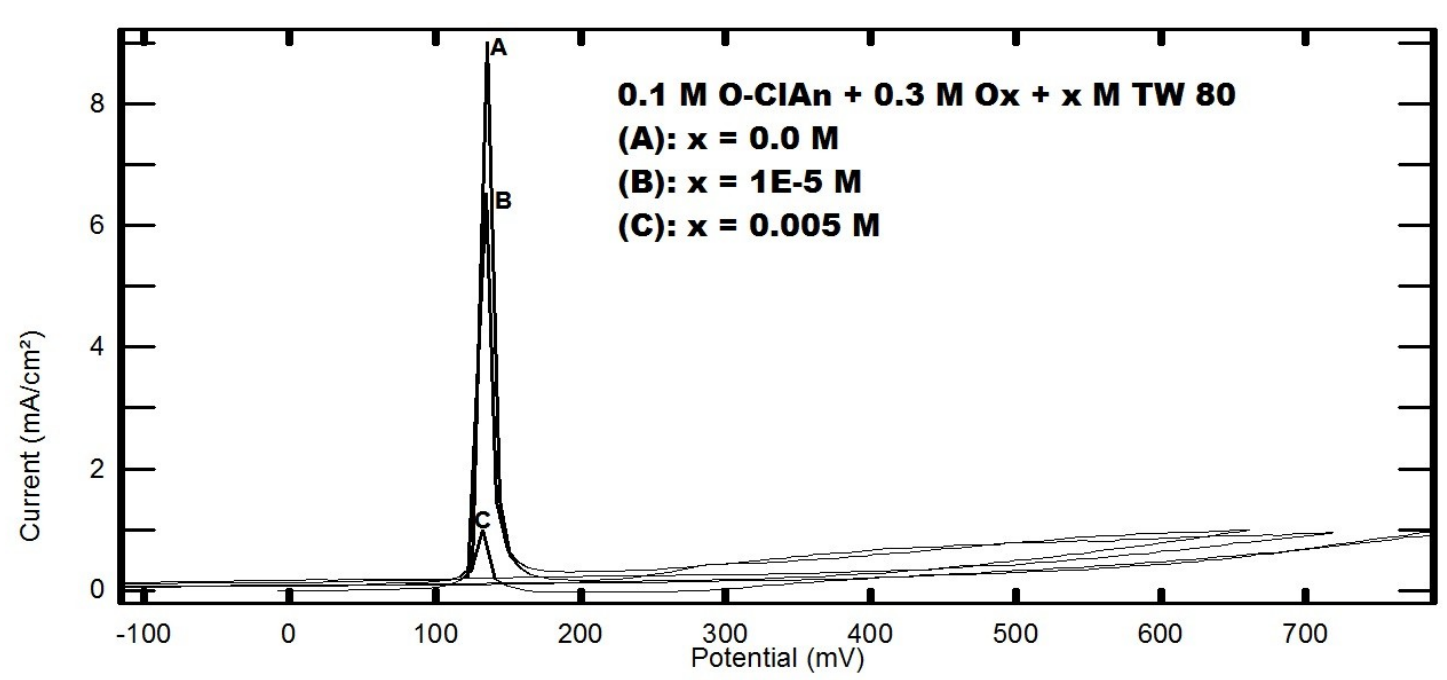

Figure 3a. The third cyclic voltammogram of steel during electropolymerization of $0.1 \mathrm{M}$ $o$-chloroaniline in $0.3 \mathrm{M}$ oxalic acid in absence and presence of different concentrations of TW 80.

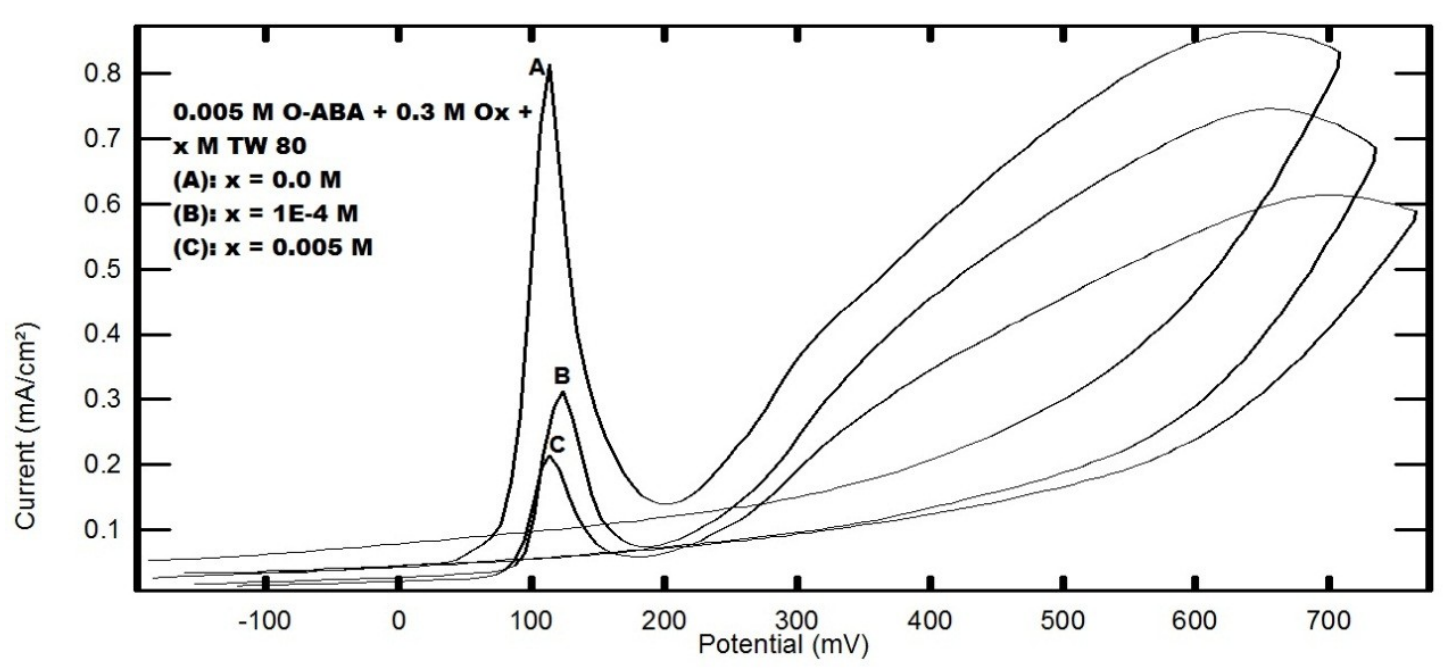

Figure 3b. The third cyclic voltammogram of steel during electroploymerization of $0.005 \mathrm{M}$ $o$-aminobenzoic acid in $0.3 \mathrm{M}$ oxalic acid in absence and presence of different concentrations of TW 80.

Figures 2, 3 indicate that the hight of the peak corresponds to the oxidation of steel is greatly dependent on the number of cycles. The height of this peak equals $47 \mathrm{~mA}$ in the first cyclic voltammogram decreases to $9 \mathrm{~mA}$ in the third cyclic voltammogram in case of $o$-chloroaniline and decreases from $72 \mathrm{~mA}$ to $0.8 \mathrm{~mA}$ in case of $o$-aminobenzoic acid. This behavior can be discussed on the basis of the formation of the polymers of $o$-chloroaniline and $o$-aminobenzoic acid in the third cycle which protect the steel surface and cause a decrease in the value of the anodic current.

Furthermore, the cyclic voltammograms in Figure 3 showed a new broad peak started at $280 \mathrm{mV}$ which is probably attributed to the oxidation of $o$-chloroaniline and $o$-aminobenzoic acid monomers to form their polymers on passivated steel. The height of this peak 
decreased by increasing of the concentration of TW 80 surfactant due to the adsorption of the surfactant molecules at the steel/solution interface.

\section{b. Inhibition of the oxidation of steel}

Figure 4a,b represent variation of the height of the peak and percentage inhibition of the oxidation of steel by Tween 80 surfactant with its concentration (from the first cycle voltammogram) in presence of a) $o$-chloroaniline and b) $o$-aminobenzoic acid.
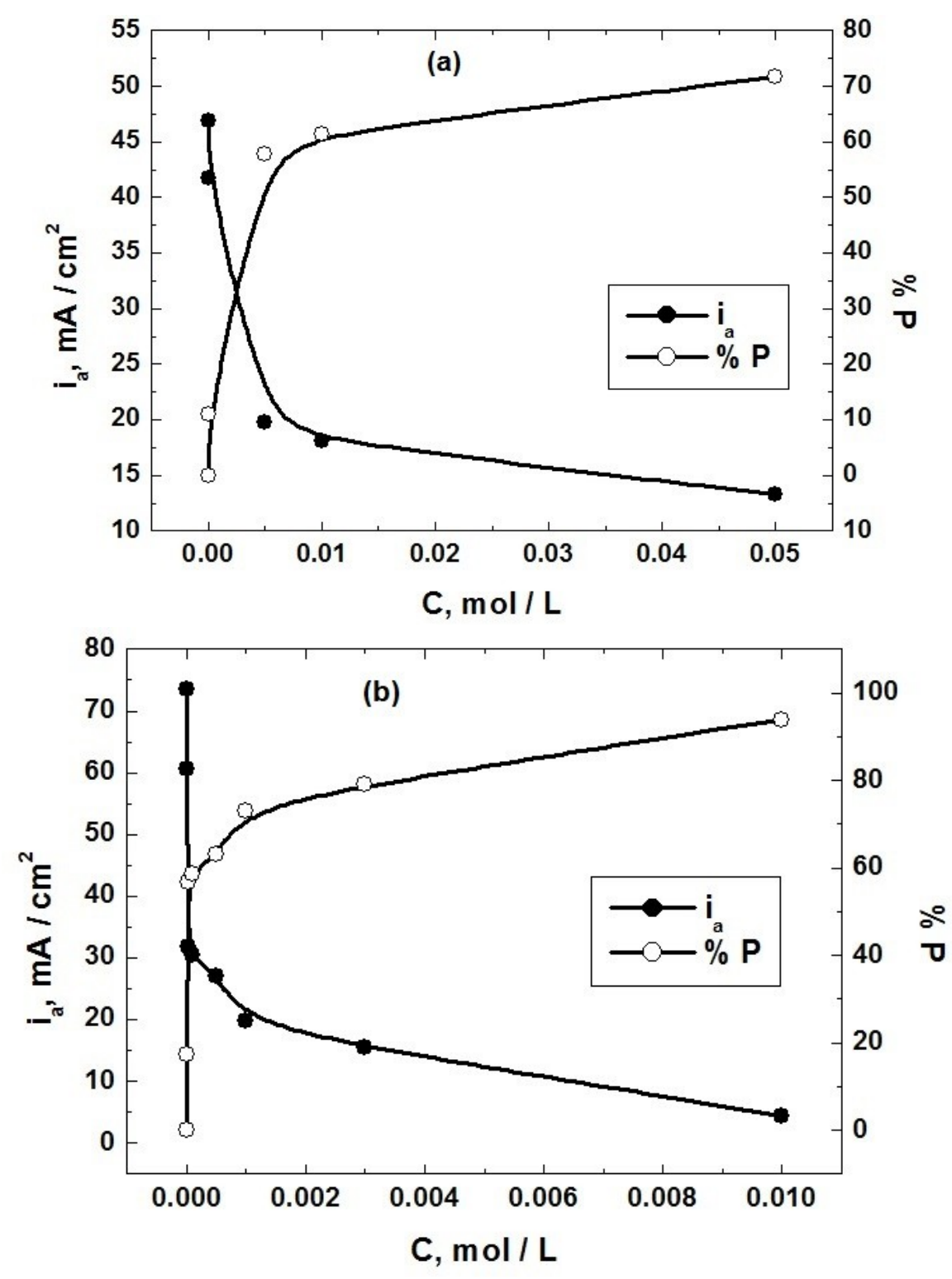

Figure 4. Variation of the height of the peak and percentage inhibition of the oxidation of steel by Tween 80 surfactant with its concentration (from the first cycle voltammogram) in presence of a) $o$-chloroaniline and b) $o$-aminobenzoic acid. 
The inhibition efficiency of Tween 80 is calculated using the equation [18]:

$$
\% P=\left[\left(i_{\mathrm{a} 0}-i_{\mathrm{a}}\right) / i_{\mathrm{a} 0}\right] \times 100
$$

Where; $i_{\mathrm{a} 0}$ and $i_{\mathrm{a}}$ are the anodic currents of the dissolution of steel in the absence and presence of certain concentration of the surfactant.

It is clear that increasing concentration of Tween 80 in the medium of electropolymerization leads to a decrease of the height of the peak and an increase of the percentage inhibition of the surfactant giving a plateau at high concentrations where is a saturation of the surface of steel with surfactant molecules.

\section{c. Adsorption of Tween 80 at the steel/solution interface}

Figure 5a,b represent the application of Langmuir adsorption isotherm to fit the experimental data obtained from the variation of the height of the peak of the oxidation of steel with the concentration of TW 80 during the electropolymerization of $o$-chloroaniline and $o$-aminobenzoic acid.

The results show that Langmuir adsorption isotherm which is given by the following equation [18] best fits the experimental results.

$$
C / \theta=1 / K+C
$$

Where $C$ is the concentration of TW $80, K$ the binding constant and $\theta$ is the surface coverage by surfactant molecules. The fitting of Langmuir isotherm indicates that the adsorption process of the surfactant molecules on the steel surface is ideal and there is no mutual interaction between its molecules. The binding constant $K$ is related to the standard free energy of adsorption $-\Delta G_{\text {ads }}^{0}$ according to the following equation [20]:

$$
K=1 / 55.5 \exp \left(-\Delta G_{\text {ads }}^{0} / R T\right)
$$

Where; $R$ is the universal gas constant, $T$ is the absolute temperature and the value 55.5 is the concentration of water in solution expressed in $\mathrm{mol} / \mathrm{L}$.

The values of $K$ obtained are 704.23 and 2414.72 which gives $\Delta G_{\text {ads }}^{0}=-26.64 \mathrm{~kJ} / \mathrm{mol}$ and $-29.76 \mathrm{~kJ} / \mathrm{mol}$ in the presence of $o$-chloroaniline or $o$-aminobenzoic acid indicating that the adsorption of TW 80 on the steel surface is mainly physical in nature. The dependence of the value of $\Delta G_{\text {ads }}^{0}$ of Tween 80 on the molecular structure of the monomer is probably due to the co-adsorption of monomer with the surfactant at the steel solution interface. Aniline derivatives are protonated in presence of $0.3 \mathrm{M}$ oxalic acid in electropolymerization medium. Several authors $[5,21,22]$ reported that the protonated aniline derivatives are adsorbed at the steel surface through bridge of counter anions (oxalate anion in this case). 

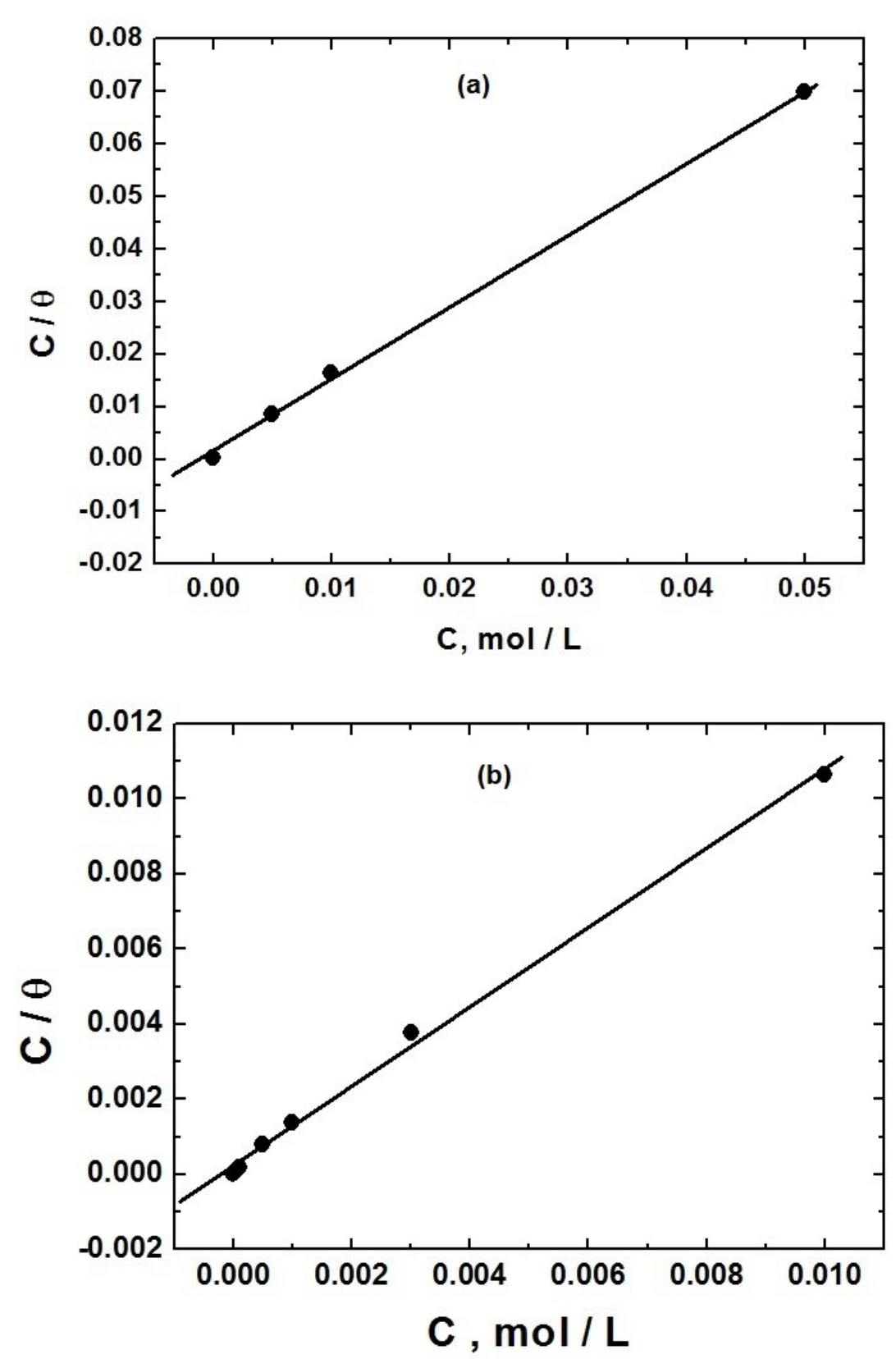

Figure 5. Application of the Langmuir isotherm to fit the data of adsorption of TW 80 on the steel surface during the electropolymerization of: a) $0.1 \mathrm{M} o$-chloroaniline and b) $0.005 \mathrm{M} o$-aminobenzoic acid in $0.3 \mathrm{M}$ oxalic acid.

B. Effect of Tween 80 surfactant on the protection efficiency of the electropolymerized coats

\section{a. Potentiodynamic polarization results}

The potentiodynamic polarization curves of the coated steel by poly $o$-chloroaniline and poly $o$-aminobenzoic acid in the aggressive environment $0.5 \mathrm{M} \mathrm{H}_{2} \mathrm{SO}_{4}$ in absence and presence of different concentrations of TW 80 are represented in Figure 6a,b. 

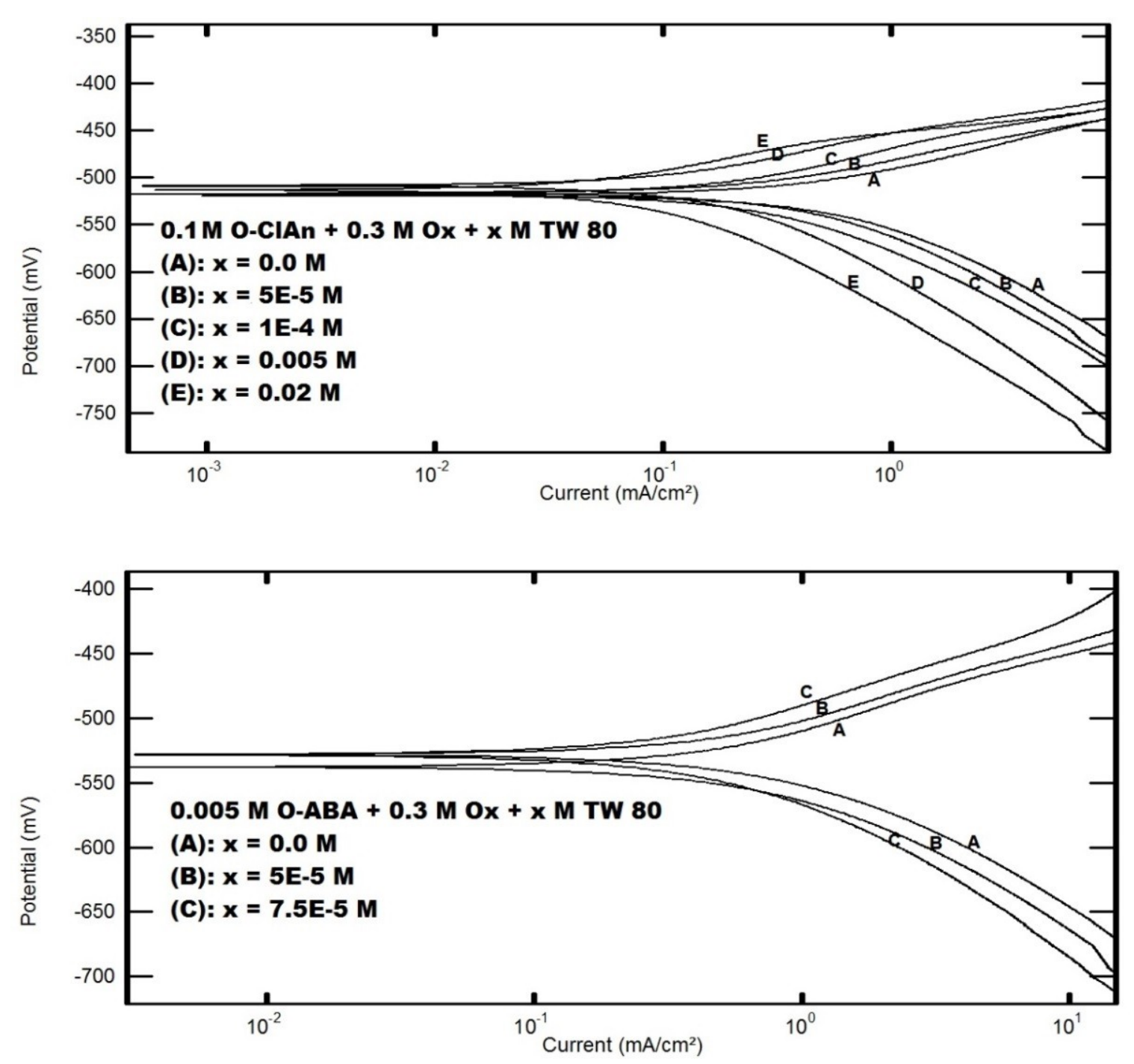

Figure 6. The potentiodynamic polarization curves for steel coated by: a) poly-ochloroaniline; b) poly $o$-aminobenzoic acid in $0.5 \mathrm{M} \mathrm{H}_{2} \mathrm{SO}_{4}$ in presence of different concentrations of Tween 80 .

Inspection of Figure $6 \mathrm{a}, \mathrm{b}$ shows that the presence of TW 80, during the electropolymerization process of each of $o$-chloroaniline and $o$-aminobenzoic acid affects both the cathodic and anodic polarization curves, indicating that the polymer coats block both the cathodic and anodic areas and inhibit the partial corrosion reactions. The values of the corrosion current density $\left(i_{\text {corr }}\right)$ were determined by the extrapolation of cathodic and anodic Tafel lines to the corrosion potential $\left(E_{\text {corr }}\right)$ and represented in Tables 1a,b.

Data in Tables 1a,b show that the corrosion current density (icorr) decreases, and the protection efficiency of the coat $(\% P)$ increases as the concentration of TW 80 is increased. $\% P$ is calculated using the equation (1). 
Table 1a. Electrochemical polarization parameters of steel coated by poly-o-chloroaniline in $0.5 \mathrm{M} \mathrm{H}_{2} \mathrm{SO}_{4}$ solution in presence of different concentrations of TW 80.

\begin{tabular}{|c|c|c|c|c|c|}
\hline \multirow{2}{*}{$\begin{array}{l}\text { Concentration of } \\
\text { surfactant }(\mathrm{mol} / \mathrm{L})\end{array}$} & \multirow{2}{*}{$\begin{array}{c}-E_{\text {corr }} \\
(\mathrm{mV} \text { vs. SCE) }\end{array}$} & $\boldsymbol{\beta}_{\mathrm{a}}$ & $\boldsymbol{\beta}_{\mathbf{c}}$ & \multirow{2}{*}{$\begin{array}{c}i_{\text {corr }} \\
\left(\mathrm{mA} / \mathbf{c m}^{2}\right)\end{array}$} & \multirow{2}{*}{$\% P$} \\
\hline & & \multicolumn{2}{|c|}{$\mathrm{mV} /$ decade } & & \\
\hline 0.0 & 502.24 & 56.698 & 125.04 & 0.65287 & 0 \\
\hline $2.5 \times 10^{-5}$ & 522.22 & 55.252 & 128.18 & 0.45883 & 29.72 \\
\hline $5.0 \times 10^{-5}$ & 497.22 & 43.573 & 137.68 & 0.39286 & 39.83 \\
\hline $1.0 \times 10^{-4}$ & 495.97 & 44.112 & 124.9 & 0.23763 & 63.60 \\
\hline $5.0 \times 10^{-3}$ & 484.53 & 36.415 & 142.31 & 0.13887 & 78.72 \\
\hline $1.0 \times 10^{-2}$ & 496.18 & 33.770 & 130.24 & 0.11998 & 81.65 \\
\hline $2.0 \times 10^{-2}$ & 483.42 & 26.633 & 138.51 & 0.07113 & 89.11 \\
\hline
\end{tabular}

Table 1b. Electrochemical polarization parameters of steel coated by poly-o-aminobenzoic acid in $0.5 \mathrm{M}$ $\mathrm{H}_{2} \mathrm{SO}_{4}$ solution in presence of different concentrations of TW 80 .

\begin{tabular}{|c|c|c|c|c|c|}
\hline \multirow{2}{*}{$\begin{array}{l}\text { Concentration of } \\
\text { surfactant }(\mathrm{mol} / \mathrm{L})\end{array}$} & \multirow{2}{*}{$\begin{array}{c}-E_{\text {corr }} \\
(\mathrm{mV} v \mathrm{vs} . \mathrm{SCE})\end{array}$} & $\boldsymbol{\beta}_{\mathbf{a}}$ & $\boldsymbol{\beta}_{\mathrm{c}}$ & \multirow{2}{*}{$\begin{array}{c}i_{\text {corr }} \\
\left(\mathrm{mA} / \mathbf{c m}^{2}\right)\end{array}$} & \multirow{2}{*}{$\% P$} \\
\hline & & \multicolumn{2}{|c|}{ mV/decade } & & \\
\hline 0.0 & 516.19 & 54.282 & 120.59 & 0.61070 & 0 \\
\hline $7.5 \times 10^{-5}$ & 509.57 & 62.226 & 129.66 & 0.47671 & 21.94 \\
\hline $1.0 \times 10^{-3}$ & 518.46 & 61.676 & 134.91 & 0.20483 & 66.46 \\
\hline $3.0 \times 10^{-3}$ & 518.17 & 56.569 & 129.81 & 0.20196 & 66.93 \\
\hline
\end{tabular}

\section{b. Electrochemical Impedance Spectroscopy results}

Figure 7 shows the Nyquist plots of coated steel (formed in oxalic acid and measured in $0.5 \mathrm{M} \mathrm{H}_{2} \mathrm{SO}_{4}$ solution) in the absence and presence of different concentrations of TW 80 surfactant during the electropolymerization process. These plots manifested only one depressed capacitive semicircle, and the diameter of the semicircle increases with increasing the surfactant concentration. The impedance spectra for different Nyquist plots were analyzed by fitting the experimental data using ZSimpWin program to a simple equivalent circuit model [18].

The values of the electrochemical parameters and the protection efficiency $(\% P)$ of the coat which polymerized in presence of different TW 80 concentrations are given in Tables 2a,b. 

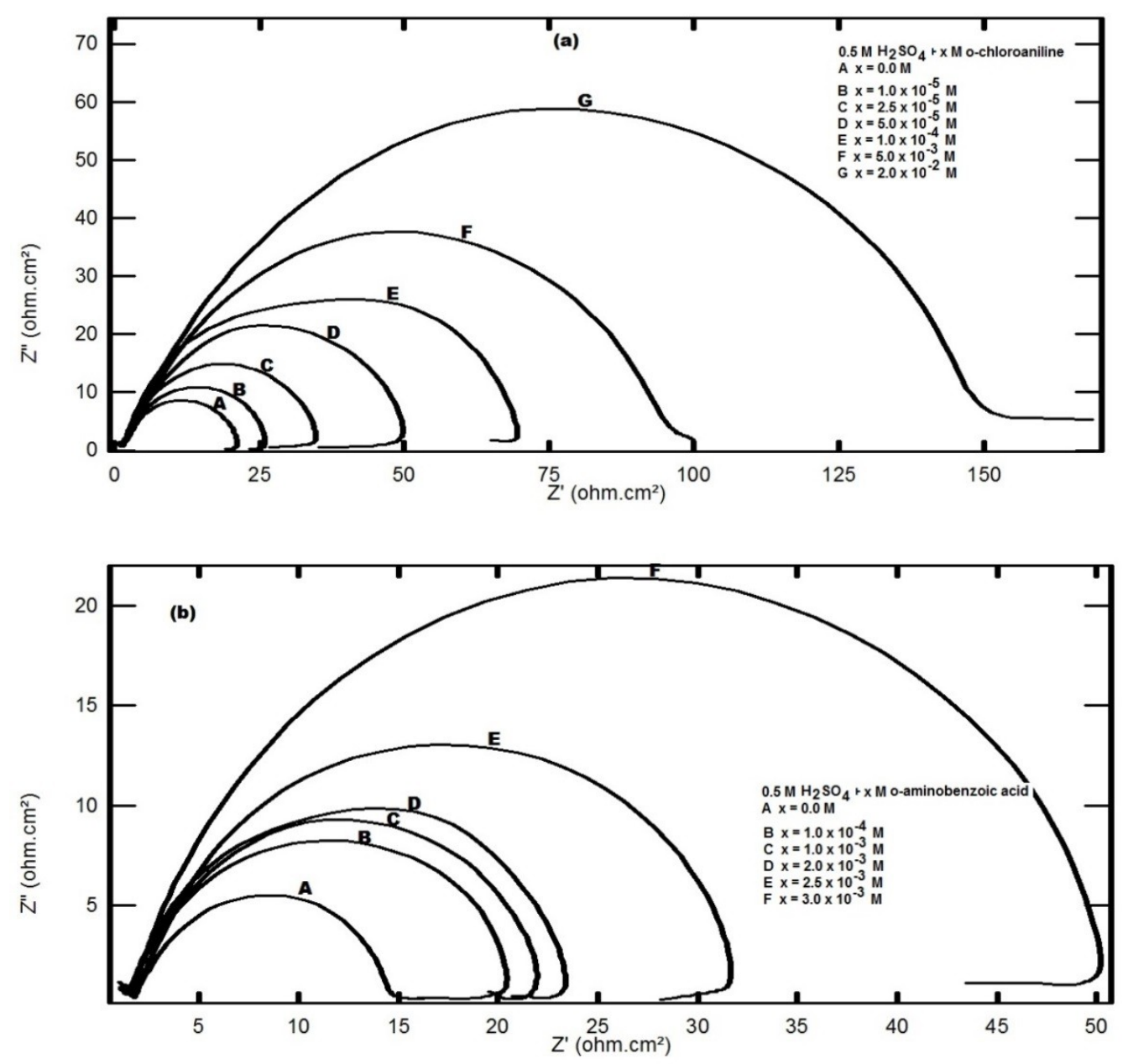

Figure 7. Nyquist plots of steel coated by poly-o-chloroaniline and poly- $O$-aminobenzoic acid in presence of different concentrations of TW 80 in $0.5 \mathrm{M} \mathrm{H}_{2} \mathrm{SO}_{4}$ solution.

Table 2a. Electrochemical impedance parameters for steel coated by poly-o-chloroaniline in $0.5 \mathrm{M} \mathrm{H}_{2} \mathrm{SO}_{4}$ solution in presence of different concentrations of TW 80.

\begin{tabular}{ccccc}
\hline $\begin{array}{c}\text { Concentration } \\
\text { of surfactant }(\mathbf{m o l} / \mathbf{L})\end{array}$ & $\begin{array}{c}\boldsymbol{R}_{\mathbf{s}} \\
\left(\mathbf{O h m} \cdot \mathbf{c m}^{\mathbf{2}}\right)\end{array}$ & $\begin{array}{c}\boldsymbol{C}_{\mathbf{d l}} \\
(\boldsymbol{\mu} \mathbf{F})\end{array}$ & $\begin{array}{c}\boldsymbol{R}_{\mathbf{c t}} \\
\left(\mathbf{O h m} \cdot \mathbf{c m}^{2}\right)\end{array}$ & $\%$ P \\
\hline 0.0 & 1.285 & 211 & 20.64 & 0.00 \\
$1.0 \times 10^{-5}$ & 1.171 & 174 & 25.79 & 19.97 \\
$2.5 \times 10^{-5}$ & 1.212 & 133 & 34.85 & 40.77 \\
$5.0 \times 10^{-5}$ & 1.234 & 110 & 50.03 & 58.74 \\
$1.0 \times 10^{-4}$ & 0.9278 & 170 & 71.05 & 70.95 \\
$5.0 \times 10^{-3}$ & 0.6681 & 108 & 95.34 & 78.35 \\
$2.0 \times 10^{-2}$ & 1.474 & 79.3 & 149.1 & 86.16 \\
\hline
\end{tabular}


Table 2b. Impedance parameters for steel coated by poly-o-aminobenzoic acid in $0.5 \mathrm{M} \mathrm{H}_{2} \mathrm{SO}_{4}$ solution in presence of different concentrations of TW 80.

\begin{tabular}{ccccc}
\hline $\begin{array}{c}\text { Concentration } \\
\text { of surfactant }(\mathbf{m o l} / \mathbf{L})\end{array}$ & $\begin{array}{c}\boldsymbol{R}_{\mathbf{s}} \\
\left(\mathbf{O h m} \cdot \mathbf{c m}^{2}\right)\end{array}$ & $\begin{array}{c}\boldsymbol{C}_{\mathbf{d l}} \\
(\boldsymbol{\mu} \mathbf{F})\end{array}$ & $\begin{array}{c}\boldsymbol{R}_{\mathbf{c t}} \\
\left(\mathbf{O h m} \cdot \mathbf{c m}^{2}\right)\end{array}$ & $\boldsymbol{\%} \boldsymbol{P}$ \\
\hline 0.0 & 1.663 & 426 & 13.02 & 0 \\
$1.0 \times 10^{-4}$ & 1.276 & 275.2 & 17.26 & 24.57 \\
$1.0 \times 10^{-3}$ & 1.126 & 185.8 & 20.24 & 35.67 \\
$2.0 \times 10^{-3}$ & 1.424 & 214.1 & 21.16 & 38.47 \\
$2.5 \times 10^{-3}$ & 1.399 & 288.5 & 31.31 & 58.42 \\
$3.0 \times 10^{-3}$ & 1.472 & 121.7 & 49.46 & 73.68 \\
\hline
\end{tabular}

The protection efficiency of the coats $(\% P)$ was calculated from impedance measurements using the following relation [18]:

$$
\% P=\left[\left(R_{\mathrm{ct}}-R_{\mathrm{ct} 0}\right) / R_{\mathrm{ct}}\right] \times 100
$$

Where; $R_{\mathrm{ct} 0}$ and $R_{\mathrm{ct}}$ are the charge transfer resistances in the absence and presence of different concentrations of TW 80 .

The data in the tables show that the values of each of $R_{\mathrm{ct}}$ and $\% P$ increase with increasing the concentration of TW 80; however, the capacity value of the electrical double layer $C_{\mathrm{dl}}$ decreases with increasing the concentration of the surfactant. These phenomena are probably due to the increase of the thickness and compactness of the coat film with increasing the concentration of TW 80 in the medium of electropolymerization process. Figure 8 shows the variation of the protection efficiency of the polymer coats for the corrosion of steel with the concentration of TW 80 surfactant.

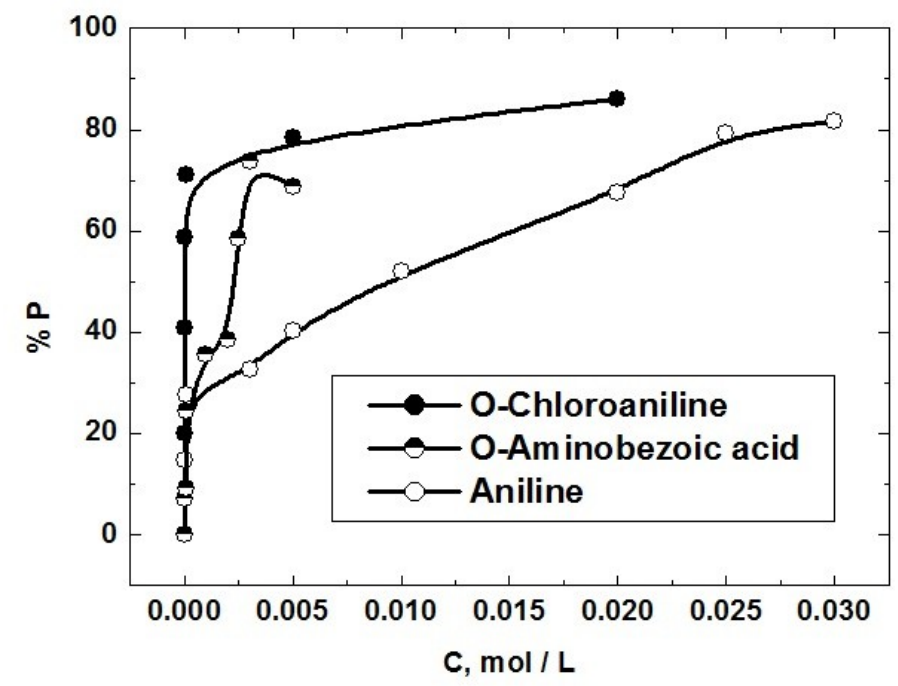

Figure 8. Variation of protection efficiency of the coat in $0.5 \mathrm{M} \mathrm{H}_{2} \mathrm{SO}_{4}$ with concentration of TW 80 from impedance measurement for the three examined compounds. [values of $(\% P)$ for aniline was taken from the previous work [18]]. 
It is clear that the coats of the polymers of the substituted compounds ( $o$-chloroaniline and $o$-aminobenzoic acid) have high protection efficiency than the polyaniline coat itself. This behavior can be discussed on the basis that both the substituent groups (carboxylic and chloro), are electron withdrawing and decrease the electron density on the amine group of the aniline molecule.

\section{c. SEM results}

Figure 9 shows the micrographs of the electrodes, recorded for bare steel and coated steel by poly-o-chloroaniline or poly-o-aminobenzoic acid in absence and presence of TW 80 surfactant.

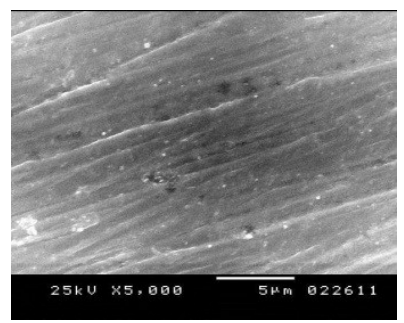

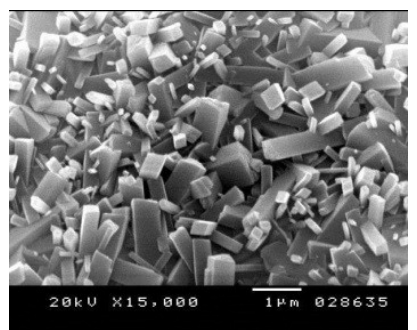

b. Steel coated by $o$-chloroaniline

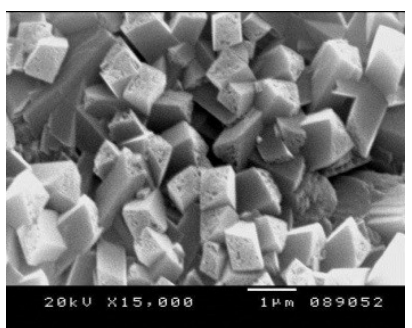

d. Steel coated by $O$-aminobenzoic acid

a. Bare steel

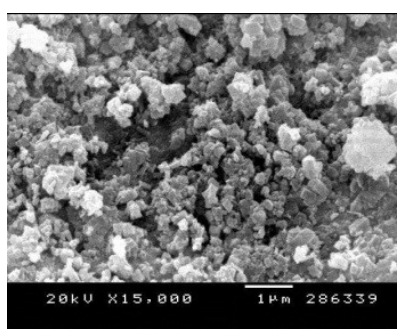

c. Steel coated by $o$-chloroaniline in presence of Tween 80

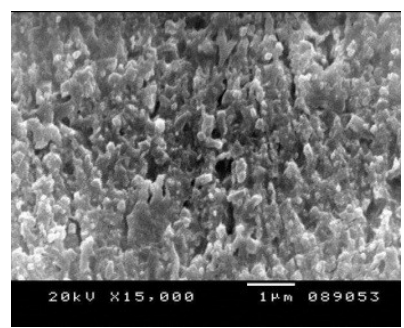

e. Steel coated by $o$-aminobenzoic acid in presence of Tween 80

Figure 9. SEM micrographs of (a) bare steel; (b) Steel coated by $o$-chloroaniline; (c) Steel coated by $o$-chloroaniline electropolymerized in presence of $0.001 \mathrm{M}$ Tween 80 ; (d) Steel coated by $o$-aminobenzoic acid; e) Steel coated by o- aminobenzoic acid in presence of $0.001 \mathrm{M}$ Tween 80 .

Figure 9a, represents SEM micrograph for bare steel which shows clear visible polishing marks on the surface. Figures $9 \mathrm{~b}$ and $9 \mathrm{~d}$, represent SEM micrographs for steel after running cyclic voltammetry experiment in $0.1 \mathrm{M} o$-chloroaniline or $0.005 \mathrm{M} o$ aminobenzoic acid and $0.3 \mathrm{M}$ oxalic acid, respectively. The crystalline structure observed 
may be due to the deposition of oxalate on the metal surface. Figures $9 \mathrm{c}$ and $9 \mathrm{e}$ represent SEM micrographs for steel after running the cyclic voltammetry experiment in $0.1 \mathrm{M} o$ chloroaniline and $0.005 \mathrm{M} o$-aminobenzoic acid respectively, in $0.3 \mathrm{M}$ oxalic acid in the presence of $0.001 \mathrm{M} \mathrm{TW} 80$. This figure shows that the morphology of each of the poly-ochloroaniline and poly-o-aminobenzoic acid is similar to the nodular structure formed by the aggregation of spherical grains which is compatible with the literature [23].

\section{Conclusions}

1. Electropolymerization of $o$-chloroaniline and $o$-aminobenzoic acid in presence of Tween 80 surfactant leads to the inhibition of the oxidation of the steel and the monomers due to the adsorption of the surfactant molecules at steel/solution interface.

2. The experimental results of the inhibition of the oxidation of steel fits Langmuir isotherm and indicated that adsorption of Tween 80 at the steel surface is mainly physical in nature.

3. Potentiodynamic polarization and electrochemical impedance results of the corrosion behavior of the coated steel electrodes indicated that the presence of Tween 80 surfactant during the polymerization process leads to the increase of the protection efficiency of the coat.

4. The results indicated that the protection efficiency of electropolymerized coats of each of $o$-chloroaniline and $o$-aminobenzoic are higher than that of polyaniline coat as a result of substituent effect.

\section{References}

1. A.B. Tadros and B.A. Abd-EI-Nabey, J. Electroanal. Chem., 1988, 24, 433.

2. A.A. El-Awady, B.A. Abd-El-Nabey and S.G. Aziz, J. Electrochem. Soc., 1992, 139, 2149.

3. A.M. Abdel-Gaber, B.A. Abd-El-Nabey, I.M. Sidahmed, A.M. El-Zayady and M. Saadawy, Corros. Sci., 2006, 48, 2765.

4. B.A. Abd-El-Nabey, O.A. Abdullatef, E. Khamis and W.A. El-Mahmody, Int. J. Electrochem. Sci., 2016, 11, 1271.

5. H.A. Fetouh, B.A. Abd-El-Nabey, Y.M. Goher and M.S. Karam, J. Electrochem., 2017 (Accepted for publication).

6. A.S. Michaels and A.R. Colville Jr, J. Phys. Chem., 1960, 64, 13.

7. D. Wang, P. Jokiel, A. Uebleis and H. Boehni, Surf. Coat. Technol., 1997, 88, 147.

8. E.P. Banczek, P.R.P. Rodrigues and I. Costa, Surf. Coat. Technol., 2006, 201, 3701.

9. R. Amini and A.A. Sarabi, Appl. Surf. Sci., 2011, 57, 7134.

10. B.A. Abd-El-Nabey, S. El-Houseiny, H.M. El-Kshlan and M.A. Abd-El-Fatah, Phys. Chem., 2017, 7, 17.

11. A.M. Fenelon and C.B. Breslin, Electrochim. Acta, 2002, 47, 4467.

12. S.R. Moraes, D.H. Vilca and A.J. Motheo, Prog. Org. Coat., 2003, 48, 28.

13. M. Kraljic, Z. Mandic and L.J. Duic, Corros. Sci., 2003, 45, 181. 
14. S. Patil, S.R. Sainkar and P.P. Patil, Appl. Surf. Sci., 2004, 225, 204.

15. V. Shinde, S.R. Sainkar and P.P. Patil, J. Phys. D: Appl. Phys., 2007, 40, 520.

16. A.M. Fenelon and C.B. Breslin, Surf. Coat. Technol., 2005, 190, 264.

17. S. Chaudhari, S.R. Sainkar and P.P. Patil, Prog. Org. Coat., 2007, 58, 54.

18. B.A. Abd-El-Nabey, O.A. Abdullatef, G.A. El-Naggar, E.A. Matter and R.M. Salman, Int. J. Electrochem. Sci., 2016, 11, 2721.

19. J.Y. Lee, C.Q. Cui, X.H. Su and M.S. Zhou, J. Electroanal. Chem., 1993, 360, 177.

20. G. Moretti, F. Guidi and G. Gion, Corros. Sci., 2004, 46, 387.

21. A.S. Fouda, Y.M. Elewady and H.K. Abd-El-Aziz, Int. J. Electrochem., 2012, 7, 10456.

22. V. Branzoi, F. Branzoi and F Golgovici, Mater. Chem. Phys., 2003, 78, 122.

23. B. Zeybek, N.O. Pekmezc and E. Kilıç, Electrochim. Acta, 2011, 56, 9277. 